EESTI NSV TEADUSTE AKADEEMIA TOIMETISED., FOOSIKA * MATEMAATIKA

ИЗВЕСТИЯ АКАДЕМИИ НАУК ЭСТОНСКОИ ССР. ФИЗИКА * МАТЕМАТИКА PROCEEDINGS OF THE ACADEMY OF SCIENCES OF THE ESTONIAN SSR.

M. РОЗМАН, В. ХИЖЖНЯКОВ

\title{
ДВУХУРОВНЕВАЯ СИСТЕМА В ПОЛЕ ДВУХ ЛАЗЕРНЫХ МОД
}

1. Задача о двухуровневой системе в резонансном мономодовом поле многие годы привлекает к себе внимание и хорошо изучена как теоретически, так и экспериментально. Поведение же указанной системы в поле нескольких либо даже двух мод изучено гораздо меньше, хотя некоторые исследования выполнены $\left[^{1}\right]$. Цель данной работы - pacсмотреть стационарные состояния двухуровневой системы в двухмодовом поле и найти ее оптические спектры. Обсуждается также случай нескольких лазерных мод одинаковой частоты. Используется метод одетых состояний $\left[{ }^{2}\right]$.

Рассмотрим вначале состояние двухуровневой системы в поле двух сильно возбужденных мод одинаковых частот $\omega_{1}=\omega_{2}=\omega_{L}$. Интенсивность их взаимодействия с системой определяется параметрами $\Gamma_{1}=\vec{d} \overrightarrow{\varepsilon_{1}} \sim d \sqrt{N_{1}}$ и $\Gamma_{2}=\vec{d} \overrightarrow{\varepsilon_{2}} \sim d \sqrt{N_{2}}$ соответственно, где $\vec{d}-$ дипольный момент перехода, $\overrightarrow{\varepsilon_{i}}-$ напряженность поля, а $N_{i}-$ число фотонов $i$-й моды $(i=1,2)$. Разница частот основного $|g\rangle$ и возбужденного $|e\rangle$ состояний ситемы равна $\omega_{0}$. Используем в качестве базиса состояния $|g\rangle\left|N_{1}-1, N_{2}+1\right\rangle,|e\rangle\left|N_{1}-1, N_{2}\right\rangle,|g\rangle\left|N_{1}, N_{2}\right\rangle,|e\rangle \mid N_{1}, N_{2}-$ $\left.-1\rangle,|g\rangle N_{1}+1, N_{2}-1\right\rangle \ldots$. В этом базисе гамильтониан рассматриваемой системы и двух мод в приближении вращающейся волны имеет вид трехдиагональной матрицы

$$
H=\left(\begin{array}{ccccccc}
\Gamma_{2} & \Delta & \Gamma_{1} & 0 & 0 & 0 \\
0 & \Gamma_{1} & -\Delta & \Gamma_{2} & 0 & 0 \\
0 & 0 & \Gamma_{2} & \Delta & \Gamma_{1} & 0 \\
. & 0 & 0 & \Gamma_{1} & -\Delta & \Gamma_{2} \\
. & 0 & . & . & : & . & .
\end{array}\right)
$$

где $\Delta=\left(\omega_{0}-\omega_{L}\right) / 2$ (за начало отсчета взята энергия $E_{\mathrm{g}}+\omega_{0}-$ $\left.-\Delta+M \omega_{L}, M=N_{1}+N_{2}\right)$. Гамильтониан (1) совпадает с гамильтонианом колебаний одномерной двухатомной цепочки (см., напр., $\left[{ }^{3}\right]$ ), собственные функции которого известны. Это позволяет сразу же найти вид искомых собственных состояний

$\left|\psi_{k, M}\right\rangle=\sum_{N}\left(C_{k, 2 N, M}|g\rangle|N, M-N\rangle+C_{k, 2 N+1, M}|e\rangle|N, M-N-1\rangle\right)$,

где

$$
\begin{aligned}
& C_{k, 2 N+1, M}=C_{1} \exp [i k(2 N+1)], \\
& C_{k, 2 N, M}=C_{2} \exp (i k \cdot 2 N),
\end{aligned}
$$

$k$ - квантовое число, аналогичное квазиимпульсу. Область изменения 
$k$ определяется условием. - $\pi / 2<k \leqslant \pi / 2$. По своему физическому смыслу $k$ соответствует скорости перехода фотонов от одной моды к другой, причем указанный поток фотонов возможен Еследствие взаймодействия между модами в присутствии двухуровневой системы.

Дальнейшие вычисления почти полностью повторяют аналогичные из теории двухатомной цепочки [ $\left.{ }^{3}\right]$. Разрешенны 2 значения энергии для каждого $M$ образуют две зоны, определяемые формулой

$$
E= \pm \sqrt{\Delta^{2}+|R|^{2}}= \pm \sqrt{\Delta^{2}+\left(\Gamma_{1}-\Gamma_{2}\right)^{2}+4 \Gamma_{1} \Gamma_{2} \cos ^{2} k}
$$

где $R=\Gamma_{1} e^{-i k}+\Gamma_{2} e^{i k}$, а амплитуды $C_{1}$ и $C_{2}$ имеют вид

$$
\begin{gathered}
C_{1}=M^{-1 / 2}(|R| / R)((E+\Delta) / 2 E)^{1 / 2}(|E| / E), \\
C_{2}=M^{-1 / 2}((E-\Delta) / 2 E)^{1 / 2}
\end{gathered}
$$

(ниже знаку. плюс в (4) соответствует $C_{1,2}^{+}$, а знаку минус $-C_{1,2}^{-}$).

2. Рассмотрим теперь оптические переходы между найденными состояниями с излучением (поглощением) фотонов других мод. Соответствующие матричные элементы дипольных переходов имеют -вид

$$
\left\langle\psi_{k, M}|\hat{d}| \psi_{k^{\prime}, M^{\prime}}\right\rangle=\delta_{k, k^{\prime}} \delta_{M, M^{\prime} \pm 1} D
$$

где $D=M d_{0} C_{1} C_{2} \exp (i k), d_{0}=\langle e|\hat{d}| g\rangle$. Скорость оптических переходов без изменения ветви в законе дисперсии - $\left|D^{++}\right|^{2}=\left|D^{--}\right|^{2}=d_{0}^{2}\left(E^{2}-\right.$ $\left.-\Delta^{2}\right) / 4 E^{2}$, для переходов с ветви плюс на ветвь минус $-\left|D^{-1}\right|^{2}=$ $=d_{0}^{2}(\mid E j+\Delta)^{2} / 4 E^{2}$, а с ветви минус на ветвь плюс $-\left|D^{-+}\right|^{2}=$ $=d_{0}^{2}(|E|-\Delta)^{2} / 4 E^{2}$. Вычисление интенсивностей соответствующих линий в спектре проведем в предположении, что состояния с разными $k$ в зонах заселены равновероятно. (Это предположение выполнено, если моды не синхронизованы; см. ниже.) Распределение заселенностей $\left(\Pi_{ \pm}\right)$по ветвям найдем из балансного уравнения $\Pi_{+}\left|D^{+-}\right|^{2}=\Pi_{-}\left|D^{-+}\right|^{2}$, $\Pi_{+}+\Pi_{-}=1$. Отсюда получаем $\Pi_{ \pm}=(|E| \pm \Delta)^{2} / 2\left(E^{2}+\Delta^{2}\right)$. Это дает для интенсивности линий на частоте $\omega_{L}$ выражение

$$
j_{0}(E)=d_{0}^{2}\left(E^{2}-\Delta^{2}\right) / 4 E^{2},
$$

а для интенсивностей их боковых сателлитов на частотах $\omega=\omega_{L} \pm$ $\pm 2|E|$

$$
j_{1}(E)=d_{0}^{2}\left(E^{2}-\Delta^{2}\right) / 8 E^{2}\left(E^{2}+\Delta^{2}\right) .
$$

Ширины линий определяются радиационными эффектами и могут быть найдены методом матрицы плотности одетых состояний $\left[{ }^{2}\right]$. В данной работе эти эффекты не учитываются.

Для нахождения суммарного спектра излучения (поглощения) необходимо знать также плотность состояний, которая в данном случае равна

$$
\begin{gathered}
\varrho(E)=\pi^{-1}|E|\left\{\left[E^{2}-\Delta^{2}-\left(\Gamma_{1}-\Gamma_{2}\right)^{2}\right]\left[\Delta^{2}+\left(\Gamma_{1}+\Gamma_{2}\right)^{2}-E^{2}\right]\right\}^{-1 / 2}, \\
E_{\min } \leqslant|E| \leqslant E_{\max }
\end{gathered}
$$

и $\mathrm{g}(E)=0,|E|<E_{\min }, \quad|E|>E_{\max }, \quad$ причем $E_{\min }=\left[\Delta^{2}+\left(\Gamma_{1}-\Gamma_{2}\right)^{2}\right]^{1 / 2}$, a $E_{\max }=\left[\Delta^{2}+\left(\Gamma_{1}+\Gamma_{2}\right)^{2}\right]^{1 / 2}$. Эта функция имеет корневые особенности на границах спектра. При $\Delta \rightarrow 0, \Gamma_{1}-\Gamma_{2} \rightarrow 0$ две средние особенности сливаются и пропадают.

Суммарный спектр состоит из центральной линии

$$
I_{0}(\omega)=\delta\left(\omega-\omega_{L}\right) \int d E j_{0}(E) \varrho(E)
$$

и крыльев 


$$
I_{1}(\omega)=\varrho\left(\left|\omega-\omega_{L}\right| / 2\right) j_{1}\left(\left|\omega-\omega_{L}\right| / 2\right) .
$$

При малых $\Delta$ (по сравнению с бо́льшим из $\Gamma_{1}$ и $\Gamma_{2}$ ) форма последних повторяет плотность состояний, так как при этом $j_{1}(E) \approx d_{0}^{2} / 81=$ const.

3. Рассмотрим предельный переход к одномодовому случаю. Его можно совершить двумя способами. 1) Положим $\Gamma_{2}=0$. Тогда будем иметь две бездисперсные зоны на частотах $\pm \Omega$, где $\Omega=\sqrt{\Delta^{2}+\Gamma_{1}^{2}}-$ частота Раби. В этом случае спектр есть хорошо известный триплет $\left[{ }^{2}\right]$. 2) Формально можно считать, что две моды получены путем канонического преобразования над базисом, в котором возбуждена одна мода. В нашем случае это будет поворот в двумерном пространстве. Так как вращательный момент до поворота равен 0, то коэффициенты при новых состояниях будут вещественными. Значит при рассматриваемом преобразовании заселяются только состояния с $k=0$ и энергиями $\pm \Omega$. Переходы между этими состояниями по-прежнему дают триплет.

4. Выше не учитывалась зависимость $\Gamma_{1}$ и $\Gamma_{2}$ от числа фотонов в модах. Однако, как отмечалось выше, возбуждению с квантовым числом $k$ можно сопоставить пакет, движущийся в пространстве $L=N_{1}-N_{2}$ с постоянной скоростью. За время $t \sim \sqrt{M} / d_{0}$ указанный пакет может переместиться на расстояние $|L| \sim M$. Тогда изменения $\Gamma_{1}$ и $\Gamma_{2}$ станут заметными. Они приведут к искажению зон на концах «решетки», что в свою очередь вызовет отражение пакета от указанных концов. В результате возникнет колебательное движение пакета и, как следствие этого, осцилляционная перекачка интенсивности от одной моды к другой. В частности в слуучае $\Delta \gg \Gamma=d_{0} \sqrt{M / 2}$, зависимость энергии возбуждения от $L$ и $k$ в области дна верхней зоны и потолка нижней зоны, согласно (4), описывается формулой

$$
E(L, k)= \pm\left(\Delta+M d_{0}^{2} k^{2} / \Delta+d_{0}^{2} L^{2} / 4 M \Delta\right) .
$$

Такой гамильтониан описывает гармонические колебания интенсивности каждой из мод с частотой $d_{0}^{2} / 2 \Delta<a_{0} / \sqrt{2 M} \sim 10-10^{6}$ сек ${ }^{-1}$ (предполагается, что $d_{0} \sim 10^{8} \mathrm{ceK}^{-1}, M \sim 10^{5}-10^{15}$ ). Отметим также, что указанные колебания можно раскачать небольшими осцилляциями интенсивности одной или обеих мод с удвоенной частотой по механизму параметрического резонанса.

5. Рассмотрим теперь случай двух мод разных частот. Здесь мы исследуем лишь наиболее простой случай мод одинаковой интенсивности с частотами, расположенными симметрично относительно частоты перехода $\omega_{0}: \omega_{1}-\omega_{0}=\omega_{0}-\omega_{2}=\Delta$. В этом случае

$$
H=\left(\begin{array}{cccccc}
2 \Delta & \Gamma & 0 & 0 & 0 \\
\Gamma & \Delta & \Gamma & 0 & 0 \\
0 & \Gamma & 0 & \Gamma & 0 \\
0 & 0 & \Gamma & -\Delta & \Gamma
\end{array}\right)
$$

(за начало отсчета взята энергия $E_{\mathrm{g}}+\omega_{1} N_{1}+\omega_{2}\left(M-N_{1}\right)$ ). Из вида гамильтониана (13) следует, что спектр энергии эквидистантен: $E_{v}=v \Delta$, $v=0, \pm 1, \ldots$. Поэтому собственные функции $H$ можно записать в виде 


$$
\left|\psi_{v, M}\right\rangle=\sum_{n=-\infty}^{\infty}\left[C_{2 n}\left(E_{v}\right)|g\rangle|n, M-n\rangle+C_{2 n+1}\left(E_{v}\right)|e\rangle|n, M-n-1\rangle\right],
$$

где

$$
C_{n}\left(E_{v}\right)=e^{i \alpha p} C_{n-p}\left(E_{v}-\Delta p\right), \quad n, p=0, \pm 1, \ldots,
$$

$e^{i \alpha p}-$ несущественный для дальнейшего фазовый множитель. Соотношение (15) позволяет выразить коэффициенты $C_{n}\left(E_{v}\right)$ через $C_{n}(0) \equiv C_{n}$. Последние, как это следует из стационарного уравнения Шредингера, удовлетворяют рекуррентному соотношению $\Gamma C_{n+1}-$ $-\Delta n c_{n}+\Gamma C_{n-1}=0$, совпадающему с рекуррентным соотношением для функций Бесселя. Поэтому

$$
C_{n}=J_{n}(2 \Gamma / \Delta)
$$

Это решение удовлетворяет условию ортонормированности.

Матричный элемент дипольного момента равен

$$
\begin{gathered}
\left\langle\psi v, M|\hat{d}| \psi v^{\prime}, M^{\prime}\right\rangle=\delta_{M, M^{\prime} \pm 1} d_{0} \sum_{n=-\infty}^{\infty} J_{2 n-v}(2 \Gamma / \Delta) J_{2 n+1-v^{\prime}}(2 \Gamma / \Delta)= \\
=1 / 2 \delta_{M, M^{\prime} \pm 1} d_{0}\left(\delta_{v+1-v^{\prime}, 0}+(-1)^{v} J_{v^{\prime}-1-v}(4 \Gamma / \Delta)\right) .
\end{gathered}
$$

Поэтому оптический спектр получается в виде:

$$
I(\omega)=\sum_{l=-\infty}^{\infty} I_{l} \delta\left(\omega-\omega_{0}+\Delta \cdot l\right) \cdot d_{0}^{2},
$$

где

$$
I_{l}=J_{l}^{2} / 4+\delta_{l, 0}\left[1 / 4+J_{0}^{2} /\left(\sum_{k=-\infty}^{\infty} J_{2 k}^{2}+1\right)\right], \quad J_{n} \equiv J_{n}(4 \Gamma / \Delta) .
$$

В пределе $\Delta \rightarrow 0, \Delta \cdot l=\omega^{\prime}=$ const огибающая спектра совпадает с аналогичным пределом результата, полученного в пункте 2. При конечных $\Delta$, таких что $\Gamma / \Delta \gg 1$, огибающая содержит осцилляции, период и амплитуда которых спадают к центру, краевые же максимумы расположены на расстоянии порядка 4 Г. Именно такой спектр наблюдался B $\left[{ }^{1}\right]$.

В заключение сделаем замечание относительно поведения двухуровневой системы в поле произвольного числа $n$ лазерных мод одинаковых частот. В этом случае нахождение энергетического спектра и собственных состояний аналогично задаче динамики $n(n-1) / 2$-мерного двухатомного кристалла. Здесь также каждому фиксированному $M=N_{1}+$ $+N_{2}+\ldots+N_{m}\left(N_{i}-\right.$ число фотонов в $i$-й моде) соответствуют две зоны, причем законы дисперсии, матричные элементы дипольного момента перехода и плотность энергетических состояний находятся аналитически.

\section{Л И Т Е Р А Т У Р А}

1. Бонч-Бруевич А. М., Вартанян Т. А., Чигирь Н. А. Ж. эксперим. и теор. физ., 77, 1899-1909 (1979).

2. Cohen-Tannoudji, C., Reynaud, S. J. Phys. B: Atom. and Mol. Phys., 10, 345-365 (1977).

3. Завт Г. С. Теория колебаний кристаллической решетки. Тарту, ТГУ, 1966.

Ннститут физики

Академии наук Эстонской ССР
Поступила в редакцию 28/II 1983 


\section{KAHENIVOOLINE SUSTEEM KAHE LASERIMOODI VÄLJAS}

On leitud kahe laserimoodi väljas asuva kahenivoolise süsteemi statsionaarsed seisundid ja optilised spektrid ning näidatud, et võrdsete sageduste korral süsteemi aatom-väli seisundid moodustavad tsoone, mis on analoogilised kaheaatomilise ühemõõtmelise ahela tsooniseisunditega. Resonantsf́luorestsentsi spektris määravad need tsoonid laserijoone satelliitide kuju. On vaadeldud võrdse intertsiivsusega, kuid erinevate sagedustega moode, mis paiknevad süsteemi siirdesageduse suhtes sümmeetriliselt, ja näidatud, et spektril on ostsilleeriv mähisjoon, mille maksimumid vähenevad keskpunkti suunas.

\section{ROZMAN, V. HIZHNYAKOV}

\section{TWO-LEVEL SYSTEM IN THE FIELD OF TWO LASER MODES}

Stationary states and optical spectra of a two-level system in the field of two laser modes are evaluated. It is shown that for equal frequency excitation the eigenstates of the atom-field system form bands analogous to those of one-dimensional two-atomic chain. These bands are manifested in resonance fluorescence spectrum in the shape of the side satellite of the laser line. The excitation by equal strength fields with different frequencies situated symmetrically with respect to the transition frequency is also studied. It is found that the envelope of the spectrum is of oscillation character representing a set of maxima converging to the transition frequency. 